Arp: The superposition of colour-magnitude diagrams from my field regions over the SMC agrees excellently with Westerlund's diagram of field stars in the SMC. I would like to point out that it was shown that the superposition of colour-magnitude diagrams for individual clusters in the SMC gives a very similar diagram. All the broad features can be recognized in these composite field colour-magnitude diagrams. The sprinkling of galactic foreground stars over these small area regions of the SMC should not interfere seriously with the interpretation of the diagram.

Eggen: There may be a spread in ages.

\title{
72. THE RED GLOBULAR-LIKE CLUSTERS IN THE MAGELLANIC CLOUDS
}

\author{
S. C. B. Gascoigne
}

Mount Stromlo Observatory

There are about 50 clusters in the Magellanic Clouds which from their spherical symmetry, integrated colours, and luminosities appear similar to the globular clusters in the Galaxy. The colour-magnitude diagrams of these clusters should give moduli for the Clouds, indications of the age and chemical composition of the clusters themselves, and perhaps some information about the evolutionary tracks of old stars generally. The first investigation of this kind was carried out by Arp on the SMC clusters NGC 361 and 419. This was followed by papers by Eggen and Sandage and by the writer on NGC 1783 in the LMC, and by Tifft (1962) on NGC 121 in the SMC. Of these four clusters only NGC 121 appeared really similar to a galactic globular cluster, the others displaying features not reproduced by any known cluster in the Galaxy. Further work was clearly needed to clarify the problems raised by these results, and a program for the systematic observation of the colour-magnitude diagrams of red clusters in the Clouds was accordingly begun here in September 1961. This contribution is a progress report on this program.

The observational material consists of blue and yellow plates taken on the 74inch telescope, calibrated by sequences observed with a photoelectric photometer on the 50-inch. In favourable conditions, that is, relative freedom from field stars, the sequences could be carried to $V=19 \cdot 0$, and, with some loss of accuracy, to $V=19 \cdot 5$. Generally, however, the program was not aimed at extremely faint stars or very high accuracy, but rather at collecting reasonably good results for as many clusters as possible. We describe here results on six clusters - Kron 3 and NGC 339 in the SMC, and NGC 1466, 2231, an anonymous companion to 2231, and NGC 2257 , all in the LMC. Because of limitations on space we can give colour-magnitude diagrams for only two of these, and in Figure 1 present instead a schematic designed to show the main features of all clusters observed to date.

We first discuss NGC 1466 and 2257, in which the Pretoria observers have discovered 46 and 27 variables respectively, mostly of short period. Their colourmagnitude diagrams are similar, both with well-defined horizontal branches, strongly concentrated towards the blue side, and equally well-marked giant branches, the termination points of which lie about $3.0 \mathrm{mag}(V)$ above the horizontal branches. In these respects NGC 1466 and 2257 resemble closely the metal-poor halo clusters, 
but there is a definite point of difference in that the giant branches of the Cloud clusters are distinctly bluer - at, say, $V=18 \cdot 0\left(M_{V} \simeq-1\right)$, their mean colours are about $B-V=0 \cdot 65$, as compared with $B-V \simeq 0.85$ for the corresponding point in halo clusters. The horizontal branches come at $V \simeq 19 \cdot 2$ and $V \simeq 19 \cdot 4$ respectively. If, on analogy with halo clusters, we attribute to these horizontal branches an absolute magnitude of $+0 \cdot 5$, the modulus of the LMC is $m-M=18 \cdot 8$.

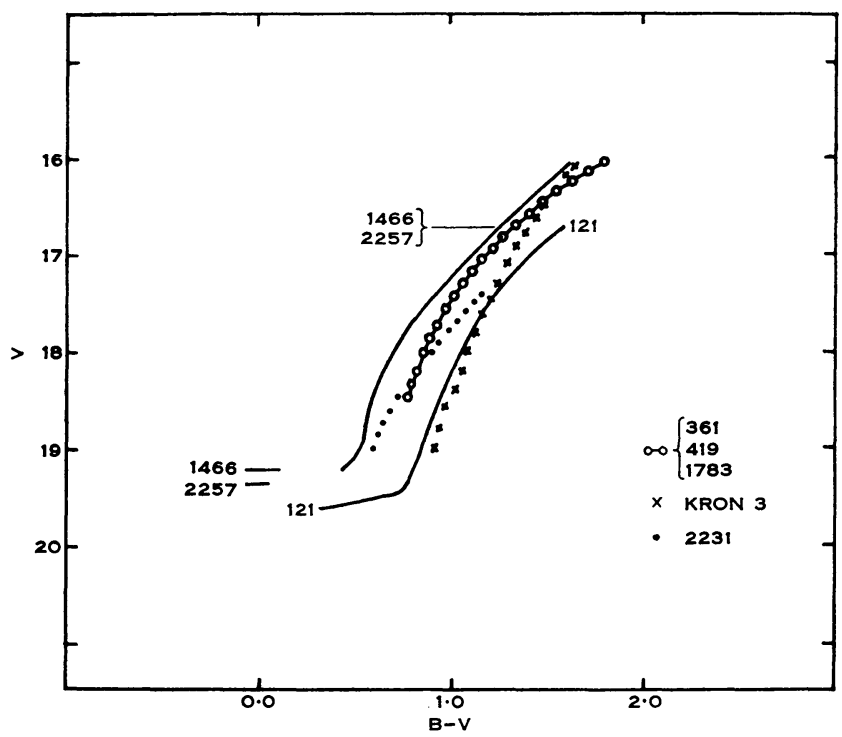

Fig. 1.-Schematic colour-magnitude arrays for most of the red clusters in the Clouds for which the data are available.

Tifft (1962) found that the variables and horizontal branch in NGC 121 came at $V=19 \cdot 6$, so that we have here an indication, though perhaps not a strong one, that the SMC is more distant than the LMC. The NGC 121 horizontal branch is concentrated to the red, and NGC 121 has clear points of difference from NGC 1466 and NGC 2257. One is tempted to suggest that the blueness of the giant branches of the two latter indicates that they are younger than the halo clusters, but this is no more than a surmise.

In a note in press (Gascoigne 1963) we have pointed out similarities between the colour-magnitude diagrams of Kron 3 and NGC 2158, the "intermediate-age" cluster resently discussed by Arp and Cuffey (1962), and have suggested that NGC 361,419 , and 1783 may also belong to this group. To this number we tentatively add NGC 339, a large, diffuse cluster situated in a part of the SMC where the richness of the background field has prevented photometry being pushed fainter than about $V=18 \cdot 8$. One of the arguments here is that none of these clusters appears to have a horizontal branch (though because of the crowded fields in which all but Kron 3 are situated it is difficult to be sure of this), whereas their integrated colours are bluer than those of almost any star in their giant branches. They must therefore have, like NGC 2158, a considerable concentration of faint (fainter than $V \simeq 21 \mathrm{mag}$ ) blue stars. These intermediate-age clusters seem to be quite common in the Clouds. 
Finally we have NGC 2231 and its companion (Fig. 2). Integrated colours and magnitudes for these are:

$\begin{array}{lccc} & V & B-V & \text { Luminosity }(V) \\ \text { NGC 2231 } & 13 \cdot 1 & 0 \cdot 54 & -5 \cdot 7 \\ \text { Companion } & 14 \cdot 3 & 0 \cdot 77 & -4 \cdot 5\end{array}$

Even without allowance for reddening NGC 2231 is very blue for a globular cluster (cf. Johnson 1959; Kron and Mayall 1960), but its most notable feature is the faintness of its giant branch, which terminates at $V=17 \cdot 0, B-V=1 \cdot 4$, about a magnitude fainter than that of its neighbour NGC 2257. NGC 2231 could of course be the more

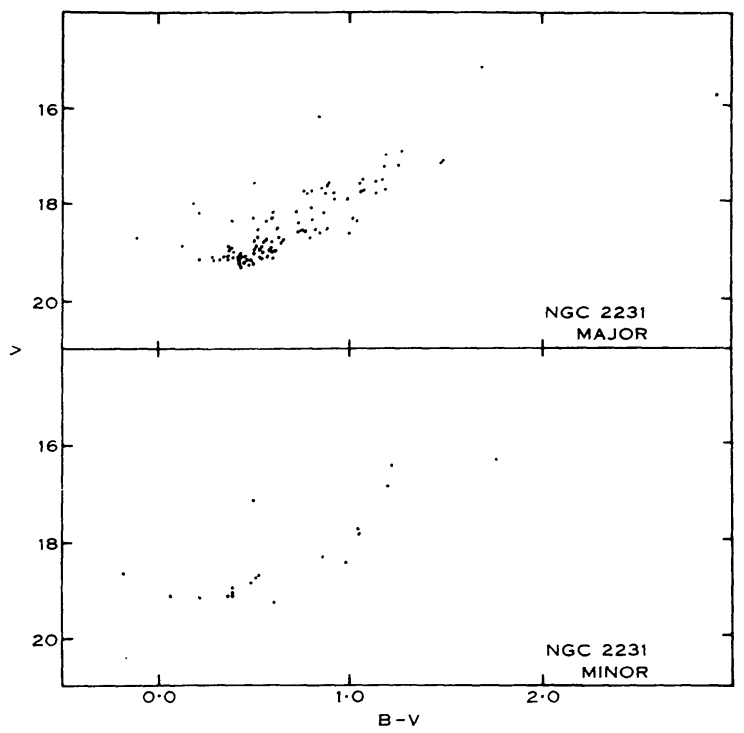

Fig. 2.-Preliminary colour-magnitude diagrams for NGC 2231 and its companion.

distant of the two, in which case it would have to be about $90 \mathrm{kpc}$ distant, as compared with $55 \mathrm{kpc}$ for NGC 2257; this seems unlikely. Another peculiarity of NGC 2231 is its number of bluish stars, especially that at $V=18 \cdot 8, B-V=-0 \cdot 05$.

The colour-magnitude diagram of the companion is even more peculiar. It has a sparsely populated red giant-like branch, with at least one bright, very red star, at $V=16 \cdot 3, B-V=1 \cdot 75$. These clusters appear to have no galactic counterpart, and on the data so far available it seems fruitless to speculate on the evolutionary processes which have led to their colour-magnitude diagrams.

To summarize then, we can now recognize in the Clouds at least three groups of red clusters:

(i) NGC 121, 1466, and 2257 resemble galactic halo clusters quite closely, though there are definite points of difference, especially with the last two;

(ii) Kron 3, NGC 339, 361, 419, and 1783 all seem similar to Arp's intermediate-age clusters, with ages somewhat less than $10^{9}$ years;

(iii) NGC 2231 and its companion appear to have no galactic counterpart, and no interpretation seems possible for these clusters at present. 
In the writer's opinion it has not yet been established that any cluster in the Clouds has properties like NGC 6356 in the Galaxy.

Clearly not all, and perhaps not any, of the Cloud clusters are as old as $10^{10}$ years. This is very different from the situation in the Galaxy where the fine halo clusters for which the requisite observations have been made appear to have quite similar ages, in the region of $10^{10}$ years, a point recently given a spectacular interpretation by Eggen, Lynden-Bell, and Sandage (1962) in terms of the sudden collapse of an originally spherical galaxy. No such phenomenon can have happened in the Clouds, where the formation of globular clusters appears to be a continuing process.

The writer would like to acknowledge the assistance he has received from Miss Flora Ogston in reducing the observations and preparing the material for publication.

\section{References}

Summaries of earlier work in this field will be found in:

ARP, H. C. (1961).-Science 134: 810.

Gascorgne, S. C. B. (1961).- Special problems in the Magellanic Clouds. In "Problems in Extragalactic Research." (Ed. G. C. McVittie.) (MacMillan: London.)

Thackeray, A. D. (1963).-Advances Astron. Astrophys. 2: (in press).

Referred to in text:

ARP, H. C., and Cuffey, J. (1962).-Ap. J. 136: 51-65.

Eggen, O. J., Lynden-Bell, D., and Sandage, A. R. (1962).-Ap. J. 136 : 748-66.

GASCOIGNE, S. C. B. (1963).-Observatory 83: 71-4.

Johnson, H. L. (1959).-Lowell Bull. 99: 117-21.

Kron, G. E., and Mayall, N. U. (1960).-A.J. $65: 581$.

TIFFT, W. G. (1963).-M.N. $125: 199-260$.

\section{Discussion}

Eggen: Some caution should probably be exercised in emphasizing a differential in the distances of the Clouds based on the position of the horizontal branch.

Tifft: If the relatively blue subgiant branch in NGC 2257 and NGC 1466, compared to NGC 121, implies that 2257 and 1466 are younger than 121, then we have additional evidence that horizontal branch and cluster-type variable luminosity increases as age decreases. This may mean we should use an $M_{V}$ calibration brighter than observed in the Galaxy to match Cloud cluster-type variables. Unfortunately this works against matching both cepheids and cluster type variables simultaneously in the Clouds with present galactic calibrations.

Woolley: I have always been interested in the question whether extremely blue objects like NGC 1818 and NGC 2004 should not be classed as globular clusters. I once discussed this with Baade who seemed to think that globular clusters should be defined to be such by their red HR diagrams. However, it has seemed to me that a much more valuable criterion is a dynamical one, namely, whether the stars have the velocity of escape or not. Now Dr. Gascoigne has produced a series of globular clusters with quite different HR diagrams, and I come back to the view that a large number of stars which have not got the velocity of escape from each other must form a globular cluster, and the largest stars must start off their lives as blue giants.

Gascoigne : I agree with the Astronomer Royal. The inference about the age and composition of the clusters depends essentially on two evolutionary tracks computed by Hoyle and Hazelgrove. Doubt has been cast on details of these by the work of Biermann and his collaborators on convection in the envelope. It needs much more work before valid inferences can be drawn from colour-magnitude diagrams. 